\title{
Chemical Kinetics in Air Plasmas at Atmospheric Pressure
}

\author{
Claudia Riccardi and Ruggero Barni \\ Department of Physics G. Occhialini, \\ University of Milano-Bicocca, Milan, \\ Italy
}

\section{Introduction}

The chapter, after a general introduction concerning plasmas produced in air at atmospheric pressure, describes the chemical kinetics happening in the plasma gas-phase, focusing on the nitrogen oxides mechanism of formation and elimination. After a general review of the chemical kinetic pattern, we discuss dielectric barrier discharges and their use for NOx remediation. In fact, research concerning the use of plasmas for the promotion of chemical reactions has emerged as one of the most prominent application field. Gas treatment aimed to toxic waste disposal or to pollutant emission reduction is an important field where plasma processing can prove to be a very effective technology. We have studied the characteristics of plasma reactors based on dielectric barrier discharges (DBD) operating in a streamer regime, as well as those based on high voltage electrical discharges operated in an intermittent spark regime. Both kind of discharges have been considered for the promotion of chemical reactions aimed to nitrogen oxides removal form a diluted gas flow. Detailed modeling of the chemical kinetics happening in an air plasma gas-phase has been investigated in our previous research. The evolution of the gas-phase composition during and after the interaction in the discharge region was calculated under different operating conditions. The results have been used to predict the NOx reduction achievable and to study the role played by the different discharge parameters in order to determine the optimal interaction between plasma and gas mixture. Comparison between streamer and spark discharges could be performed too.

\section{Discharges at atmospheric pressure}

Let's consider a system where at least two electrodes (conductive, in general metal surfaces) are separated by a gap filled with air at atmospheric pressure. Then one of the electrode electrical potential is increased respect to the second, which we could suppose, without any lack of generality to be grounded. An electric field arises in the gas gap, whose magnitude and space distribution depend on the potential applied and the geometry of the system. At large enough values of the field a discharge could spark in the gas. When an electrical breakdown happens in atmospheric pressure air the discharge quickly evolves towards to a condition allowing large electric current flows and strong heating of the gas. Self-sustaining discharges at such high pressure could not develop as the usual glow regime, since heating 
and ionization instability leads to the contraction of the discharge electrical current channel and, if the system could sustain it, the discharge evolves to a so called arc. Under such conditions the air in the gap becomes very hot, reaching a few thousands of $\mathrm{K}$ and electrical current could exceed hundreds Ampers (Raizer, 1991).

These kinds of discharges have interesting chemical kinetics in their own, being almost thermal equilibrium systems, but their behaviour as chemical kinetics reactors bears more resemblances with that of a hot gas than of a plasma. Moreover such devices require very large power and the high temperatures imply a considerable stress of the materials used, which somewhat limits their use as processing tools in material chemistry.

\subsection{Corona and radiofrequency discharges}

More interesting from the point of view of plasma chemical kinetics is another kind of discharge, the so called corona. However corona discharges occur only whether the electric field is sharply non-uniform in space, which means that the electrode setup should reflect such condition. The easiest way to accomplish this is when one of the electrode size is much smaller than the inter-electrode or gap distance. Point or wire electrodes are the best instances of that. Corona are self-sustaining discharges, producing plasmas in steady conditions and burning in a stable manner for quite long periods (Raizer, 1991). Then chemical kinetics have time to evolve and an interesting pattern of radical formation and oxidation could develop in the air of the gap. On the other hand, corona discharges are inherently disuniform in space, requiring geometry to be taken into account and complicating the task of modeling. The result is, in a sense, to obscure partially the true chemical kinetics effects. Corona discharges draw relatively small electrical currents and plasma density is usually small too, making their reactivity quite low. Then their use in material processing is somewhat limited, even if, mainly in non specialized literature, the term corona is often employed and abused for other kind of discharges and setups. In the following sections we will discuss briefly the chemical kinetics associated with their plasmas. Although the use of an oscillating voltage as supply could make several differences in the gas breakdown and in the discharge formation, there are striking similarities in the plasma state which is produced. Indeed at the somewhat large density of an atmospheric pressure gas the oscillation frequency is order of magnitude smaller than the elastic collision frequency (in air at STP this is about $3 \mathrm{THz}$ or $3 \times 10^{12} \mathrm{~Hz}$ ). Under such conditions averaging

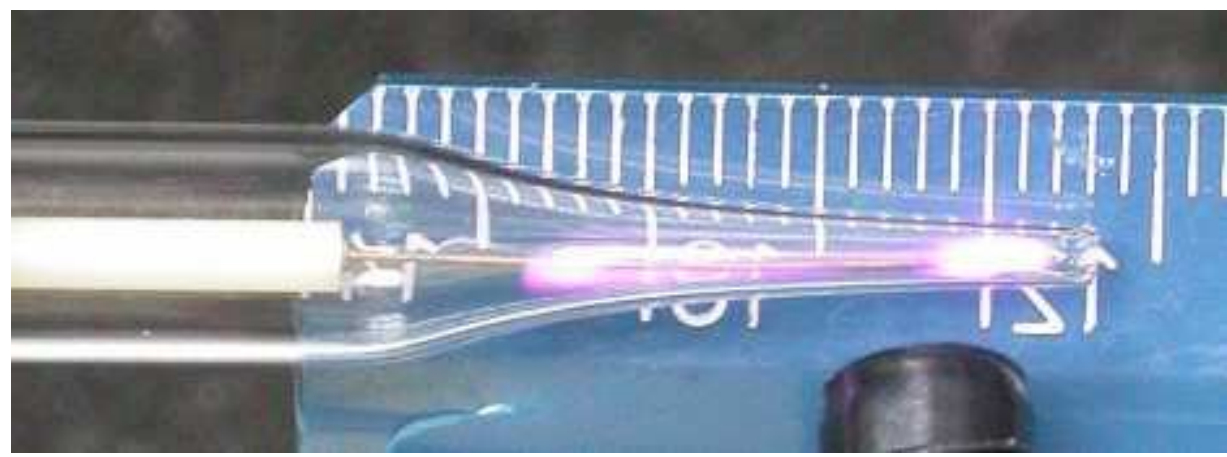

Fig. 2.1.1. A radiofrequency atmospheric pressure air plasma produced by a plasma needle. 
occurs and a steady plasma state is formed, independent on time and, in first approximation, determined only by the effective, i.e. rms, electric field value and not from the wave frequency (Raizer, 1991). Then modeling as well as the pattern of the chemical kinetics associated with these plasmas could be grasped from the trends observed in d.c. corona discharges. The oscillating electric field however prevent most of the charges to be lost at the walls or the electrodes by drifts alone and the plasma state is less influenced by badly known parameters linked to electrode surface and material properties as in general it happens in d.c. configurations. This make the realization of suitable plasma sources in form of plasma jets, pencils or needles much easier and user-friendly (Mariotti, 2010).

\subsection{Dielectric barrier discharges}

The main characteristic of a Dielectric Barrier Discharge (DBD) device lays in the setup of the electrodes. In particular the setup includes the presence of a dielectric layer within the discharge gap distance $\mathrm{d}$ (in general in the 0.1-5 $\mathrm{mm}$ range for an atmospheric plasma) insulating at least one of the electrodes. The dielectric presence forbids the flow of a direct current in the gap, preventing the formation of arcs or sparks, as well as of a steady plasma state. To sustain the discharge an a.c. voltage or a pulsed one is applied to the electrodes at a frequency ranging from several hundreds of hertz to a few hundreds of $\mathrm{kHz}$. While many different kinds of DBD exist, in general, for a such high value of the product pd of gas pressure $\mathrm{p}$ and the electrode gap width $\mathrm{d}$, the discharge operates in a streamer regime (Raizer, 1991). Just to make a little example, let's consider a discharge gap d of $1 \mathrm{~mm}$ supplied by an HV voltage of $5 \mathrm{kV}$, which makes an electric field of $5 \mathrm{MV} / \mathrm{m}$ whether it would be uniform. Using the data reported in Fig. 2.2.1 it easy to see that the ionization length is well below $0.1 \mathrm{~mm}$. Actually the product $\mathrm{a} \cdot \mathrm{d}$ turns out 21.3 exceeding the threshold value of 18-20, known as the Meek criterion, for streamer discharge onset (Raizer, 1991).

But what characterises most the steamer regime? These discharges are built up of many repeated microdischarges, each leading to the formation of narrow discharge filament (with an electron density in the range $1012-10^{17} \mathrm{~cm}^{-3}$ ) of some nanoseconds duration (Kogelschatz, 2003). In particular a plane electrode DBD at near atmospheric pressure which operates in streamer regime, under an a.c.voltage supply displays an uniform glow filling the gap. At a closer inspection the glow reveals itself as built up with a large number of current filaments appearing through the gap at seemingly random locations and not exactly at the same time during an active phase which happens each voltage half-cycle (Kogelschatz, 2003). The uniformity is just brought in by the high rate of repetition and superposition of such microdischarges. Depending on the operating conditions, at each half-cycle a new streamer head could propagate in the opposite direction starting from the same location when the voltage is reversed. On the other hand, during the active phase, the streamers cannot propagate on the same path, being inhibited by the space charge accumulated on the dielectric surfaces. This has important consequences on the chemical kinetics and its modeling since streamers can be considered as isolated and repeating at frequency which is the double of the a.c. voltage frequency. Another key feature is that, at least in small gaps like the one we are considering, they appear to be very thin (radius $\mathrm{R} \sim 100 \mu \mathrm{m}$ ) while their average distance is much larger (a few $\mathrm{mm}$, of the order of the gap width) as discussed in literature (Raizer, 1991). So, as a first approximation one could chose to simulate the chemical kinetics induced in a single isolated streamer and its repetition at a fixed frequency. The results of this approach are examined in the following. 


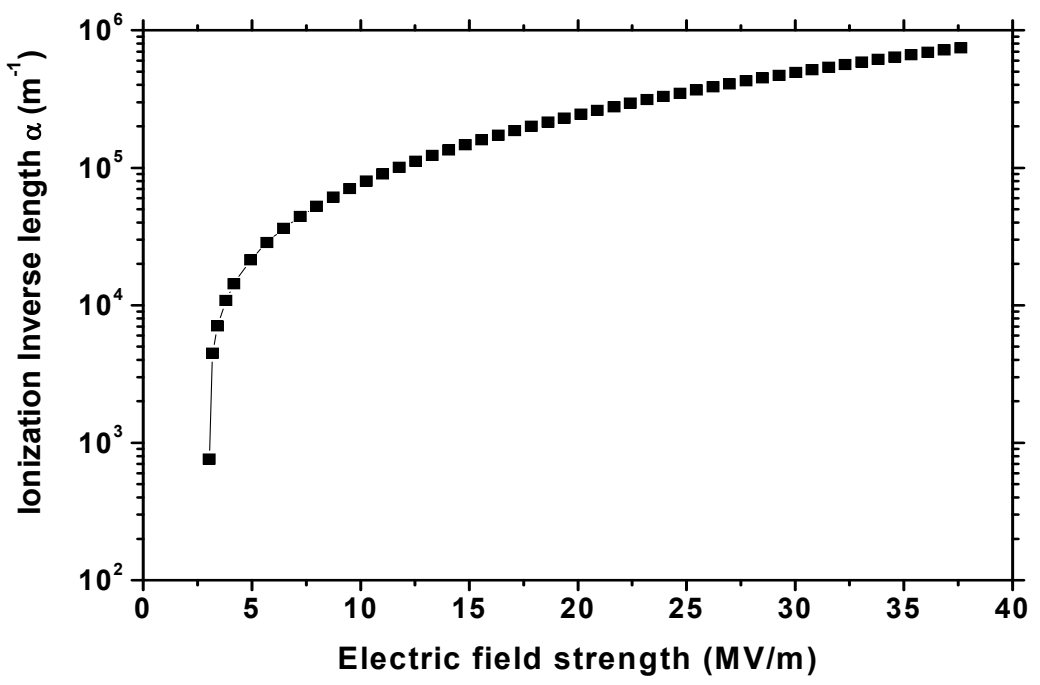

Fig. 2.2.1. The ionization inverse length coefficient in atmosperic pressure air

Several models (see for instance Kulikovsky, 1997-1998) and a few experimental observations (Malik, 2008) of streamer development and propagation exist by now. Despite many differences, the overall picture appears to be the same in all kind of DBDs. The microdischarges develops in three steps. After a phase of charge accumulation in the head, coming from previously deposited charges or from electron multiplication (Raizer 1991), the stremer head starts moving towards cathode. Streamer propagation is very fast $\left(\mathrm{v} \sim 10^{5}-10^{6}\right.$ $\mathrm{m} / \mathrm{s}$ ) and is sustained by the electron avalanche multiplication in the strong field ahead of the streamer $\left(\mathrm{E}_{\max } \sim 5-15 \mathrm{MV} / \mathrm{m}\right)$. After the head has reached the cathode, spreading its charge on the dielectric surface, in the gap a discharge filament is left. The streamer body consists of a thin channel of weakly ionized gas, almost quasi-neutral $\left(\mathrm{n}+\sim \mathrm{n}-\sim 10^{13}-10^{16}\right.$ $\mathrm{cm}^{-3}$ ) and driven by a small or just vanishing electric field. After the streamer head reaches the dielectric barrier insulating the cathode, the channel allows the flow of a limited discharge current, which is rapidly quenched by the space charge accumulating at the dielectric surface. At high pressures electrons moves along the electric field at the drift velocity and almost instantaneously (the corresponding relaxation times for velocity and energy, dictated by collisions with neutrals, are $\tau \sim 0.3 \mathrm{ps}, \tau_{\mathrm{E}} \sim 50 \mathrm{ps}$ ) reach a mean energy determined by the local amplitude of the field. On the other hand ions inertia keeps them almost frozen and substantially cold, that is at the set temperature. At a fixed position along the streamer path, the electric field rises quickly as the streamer head is approaching and then decay almost as fast to the much smaller value it assumes in the streamer channel. This has important consequences on the chemical kinetics and its modeling too. Indeed the high energy bond-dissociation and ionization processes happens only through electron impact interactions, since neutral and ions are mostly cold. Then a simple cold plasma approximation is valuable. Moreover tha active phase happens only in a very short time when the streamer head is propagating. Since propagation is so fast, chemical kinetics could be considered freezed and happening independently at each position along the streamer channel (Siliprandi, 2008). 


\section{Chemical kinetics in atmospheric pressure air plasmas}

In numerous problems of plasma chemistry, electro-physics, gas lasers physics and atmospheric physics a detailed consideration of the kinetics of non-equilibrium plasmachemical processes in nitrogen-oxygen mixtures is essential (Raffaele-Addamo, 2003; Esena, 2005). Another relevant topic concerns the need to examine the ecological consequences of projects involving a prolonged use of powerful electromagnetic radiation in the atmosphere. Channels determining the balance of neutral and charged particles in a non-equilibrium plasma are highly diverse, making plasma chemical kinetics vastly different from that of an ordinary hot gas. In plasmas, the main chemical processes proceed on the basis of reactions with nitrogen and oxygen atoms being formed by electron impact dissociation of the initial molecules. In some cases, electronically excited molecules play an important role, as for instance, the influence of oxygen molecules in the state a' on the process of ozone formation. This makes relevant any work undertaken in an attempt to form a kinetic scheme of nonequilibrium discharge in nitrogen-oxygen mixtures (Kossyi, 1992).

\subsection{Modeling of the air kinetics in dielectric barrier discharges}

As discussed above, a sensible starting point to simulate the chemical kinetics induced in a DBD is to study what happens in a single isolated streamer and its repetition at a fixed frequency. For the sake of simplicity we choose to discuss dry air. However the validity of the model can be extended to general electrodes configuration and more extensively to any plasma devices operating in a streamer discharge mode. The inherent difficulty encountered in developing a model for air discharges at high pressure is that one must solve a highly nonlinear coupled set of differential equations which may be both spatially and temporally dependent. In DBDs, moreover, the time scales involved span many order of magnitude, from the very fast connected with streamer formation and propagation to the very slow connected with ion recombination, chemical kinetics of neutral species and diffusion. So several approximation are worthy in order to make tractable the problem. From the chemical kinetics point of view, the process can be seen as consisting of an almost instantaneous phase during which energetic electrons produce mostly ions and atomic radicals, followed by a phase in which electrons cool down almost instantaneously and reach the set temperature or a temperature so low, that further dissociations are stopped. In the latter phase, slow chemical reactions between neutral and charged species happen in the gas phase, while they diffuse outside the streamer channel. Although existing models do predict that the shape as well as the peak value of the electric field pulse depends on the position along the streamer path, the differences are not large, apart from the region near the dielectric surfaces (Kulikovsky, 1997). As a first approximation, one could neglect such differences and thus let's suppose uniformity along the streamer path. From the mathematical point of view, then the model formulation used treats the streamer channel as a cylinder, with a circular cross-section. The radial profile is determined by diffusion and the only parameter describing evolution is time. The actual value of the streamer radius is treated as a free parameter of the simulation. We assume also a square pulse shape for the time evolution of the electric field ahead of the streamer. The pulse is therefore characterized by the time length (here 2 ns, based on the model in (Kulikovsky, 1998)) and by the peak electric field $E_{\max }$. Although it appears as a very rough approximation, it embodies all the relevant dynamics for our aims. 
In building our model and performing numerical simulations we have found more sensible to employ two different parameters, which are related to those describing the electric field ahead of the streamer. The first one, arises naturally when we have observed that since the electron density rises almost exponentially as the streamer develops, the total amount of radicals produced by electron impact does not depend strongly on the time length of the pulse or on the initial electron density but only on the actual value reached by the electron density at the end of the pulse. We have treated the value reached by the electron density ne at the end of the streamer passing as the relevant parameter of the simulation. As reference we consider a density of $10^{15} \mathrm{~cm}^{-3}$ (Braun, 1991; Kulikovsky, 1998). However we have varied $\mathrm{n}_{\mathrm{e}}$ in a broad range in order to investigate how it can affect the gas-phase chemical kinetics.

After imposing that the electron density reached in the streamer channel after its formation were the same, we also checked that the length of the electric field pulse does not make any significant difference in a broad range from 0.2 to $5 \mathrm{~ns}$. This range is enough to cover the different streamer head velocities reported in DBDs (Raizer, 1991). As it could be guessed, most of the chemical kinetics is simply frozen during such fast times and the total production of species through electron impact reactions during the pulse depends on the electron reaction rates (influenced by the electric field amplitude) and on the electron density which is reached at the end of the pulse rather than on the pulse length.

As for the second parameters, the electric field strength $E_{\max }$, we have found more sensible to employ the electron temperature instead. In fact it was simpler to express the electron rate constants, calculated from their cross-sections and evaluated assuming a Maxwellian energy distribution function for electrons, described by a single parameter, their temperature $T_{e}$.

Then it was possible to collect reaction rates in form of simple functions of a single parameters with Arrhenius formulas, which are quite common in literature and databases of chemical kinetics and reaction rates. The use of electron temperature as a parameter does not prevent the capability to make comparison with other existing models, since it could be related, in a one to one correspondence, with experimental informations on the streamer electric field. Indeed electron temperature is trivially connected with the mean electron energy, which is determined by the local electric field in the Boltzmann equation (Raizer, 1991). This is sufficient to make straightforward a direct comparison between this simulation and other existing ones or experimental data. In the following we considered as reference an electron temperature value of $4 \mathrm{eV}$ (Kulikovsky, 1998).

Under such simplified description of the streamer development, we could model the subsequent evolution of the gas phase in a standard way, using the continuity equations for each chemical species and solving a system of mono-dimensional first-order differential equations easily and quickly tackled by numerical integration (Riccardi, 2000). From a chemical engineering point of view, indeed it means that the model can be formulated as a well-mixed reactor (Benson, 1982). The gas-phase composition in the reactor is determined by the chemical reactions among the reactive species and the transport processes. The time evolution of the concentration of the different $\mathrm{N}$ species in the gas phase is determined by integrating each balance equation for the density $n_{k}$ of the $k^{\text {th }}$ species:

$$
\frac{d n_{k}}{d t}=\sum_{i<j=1}^{N} K(i+j \rightarrow k) n_{i} n_{j}-\sum_{i, j=1}^{N} K(k+i \rightarrow j) n_{k} n_{i}+D_{k} \nabla^{2} n_{k}
$$


where $\mathrm{K}$ are the reaction rates for the gas phase reactions and $\mathrm{D}$ is the diffusion coefficient. Due to the supposed uniformity, diffusion along the streamer axis is neglected. Adsorption on the dielectric barrier is included but, because of the much smaller extension of the streamer radius respect to the discharge gap, it turns out to be negligible. The choice of species included in the model was based on existing experimental information based on emission spectroscopy or mass spectroscopy. A sub-set of 8 neutral and 9 charged species was included in a preliminary study. Then the code was updated to include 20 neutral and excited and 12 ionic states.

As already discussed, to simulate DBD plasmas a cold plasma approximation is suitable. So reaction rates for ion as well as neutral species are evaluated at the set temperature, that is $300 \mathrm{~K}$. The effect of larger temperatures is addressed below. Since thermal energy is much smaller than the molecular bond energy, the initial step in the decomposition is presumed to involve mainly electron-impact dissociation. Electron-impact cross-sections used in this study were obtained from literature collected in (Riccardi, 2001; Barni, 2005), apart from attachment rates which are taken from recent experiments. Rate constants have been evaluated assuming a Maxwellian energy distribution function for electron, described by a single parameter, their temperature Te. Rate constants for neutral gas-phase, charge exchange and ion recombination reactions have been taken from literature referenced in (Riccardi, 2001; Guerra 1997) or estimated based on approximating techniques (Benson, 1982). In this report we assume a substantial vibrational excitation of nitrogen molecules, which allows direct dissociation from atomic O radicals (Guerra, 1997). A total of 121 reactions have been taken into account in the simulation. The diffusion loss rates are estimated by using a formulation due to (Chantry, 1987) in cylindrical coordinates. The radial density profile of the streamer is approximated in term of the lowest order normal mode and is integrated away. This formulation allows substituting the Laplacian operator with an effective diffusion length and makes the system of balance equations only time dependent. Diffusion of air from the outside the streamer is also included as a source term. Ion diffusion loss rates was corrected by enforcing the plasma quasi-neutrality, following an approach proposed for low pressure electronegative plasmas (Lichtenberg, 2000). Balance equations containing all the processes described above form a system of coupled differential equations which has been integrated to obtain the time evolution by using an adaptive Runge-Kutta routine. Results from the calculations are discussed below and have been partially presented in literature (Barni, 2005; Barni, 2010).

\subsection{Chemical kinetics pattern in air}

As a first step we start discussing the temporal evolution of the discharge gas phase using a sort of reference values for the free parameters. That is electron temperature was $4 \mathrm{eV}$, electron density reached in the microdischarge channel is $2 \times 10^{15} \mathrm{~cm}^{-3}$ in a time length of $2 \mathrm{~ns}$ and the streamer radius was $100 \mu \mathrm{m}$. At first, we neglect diffusion losses, in order to look directly the evolution pattern of the chemical kinetics alone. Neutral as well charged species densities are displayed as a function of time respectively in figures on a log-log scale.

As expected, the charged phase shows a very fast increase, almost exponential, in almost all species, as the streamer gets formed. Afterwards, as diffusion is not included, the system evolves under recombination alone, giving the overall decreasing trend as the inverse of time (that is linear in the log-log plot). The composition however displays some important 


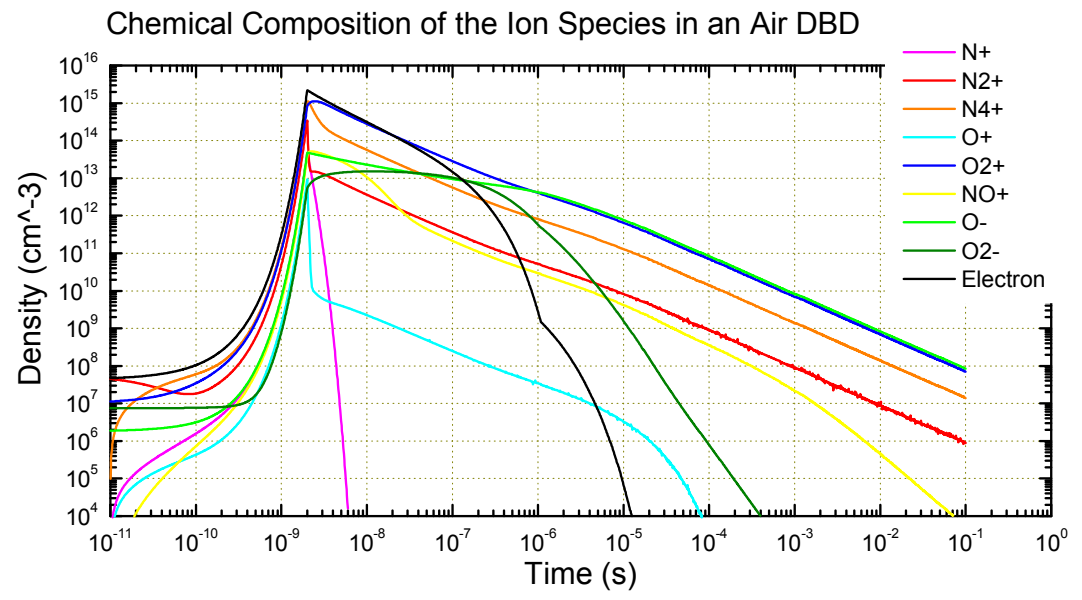

Fig. 3.2.1. Evolution of the charged density in the gas-phase of a streamer in an air DBD.

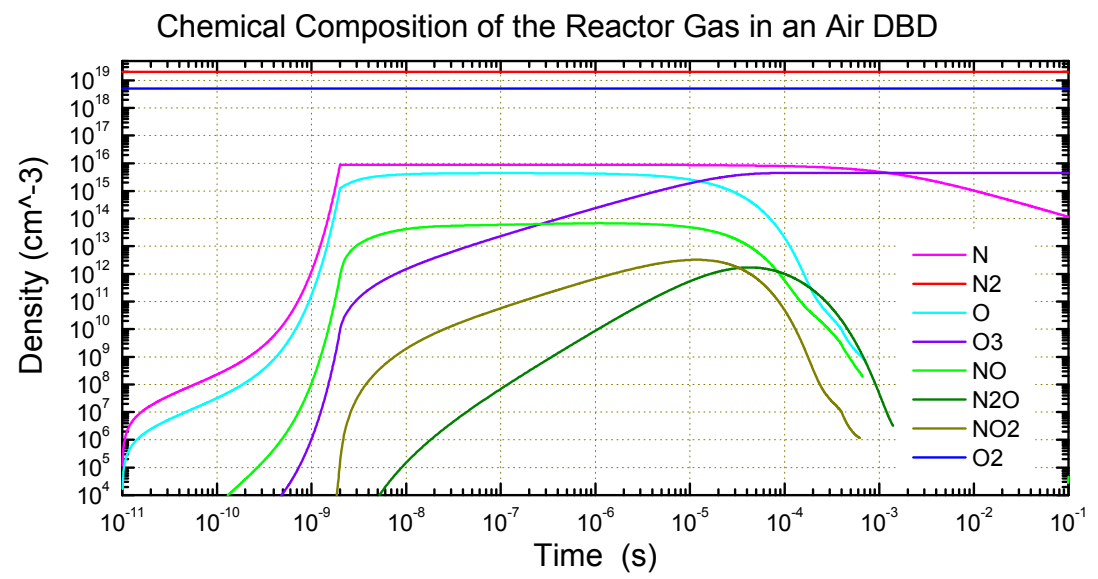

Fig. 3.2.2. Time evolution of the neutral species density in the gas-phase of a streamer in an air DBD. After a brief transient due to streamer formation, the neutral gas-phase reaches an equilibrium with the formation of ozone in a few ms.

changes. First atomic ions are removed immediately due to charge exchange reactions on nitrogen and oxygen molecules. Then electrons are lost, due to prevalence of attachment for cold electrons in the streamer channel. Their evolution reflects the shift between atomic and molecular oxygen negative ions. Later on, a sort of equilibrium in the positive ion composition is reached. It reflects the strength of interactions with main neutral molecules. The major ions are then negative atomic oxygen $\mathrm{O}^{-}$and positive molecular oxygen ion $\mathrm{O}_{2}{ }^{+}$, with traces of nitrogen and nitrogen oxides ions. As for the neutral species, during the time length needed for streamer development mainly atomic $\mathrm{N}$ and $\mathrm{O}$ species are created, from electron impact-dissociation (the former more than the latter at such an electron 
temperature). NO production is quite fast, the fastest among other molecules, but even so its density stays under $0.1 \%$ by the end of the streamer formation time. Approach to equilibrium is much slower. Between 10 and $100 \mu$ s oxygen atoms decline and molecules accumulate, with ozone already dominating. With the disappearance of $\mathrm{O}$, all nitrogen oxides decline, leaving only ozone (besides air) in the gas phase. Nitrogen atoms are much less reactive and are removed only late, in tens ms, mainly through recombination to $\mathrm{N}_{2}$, as their linear decay in log-log plot clearly reveals. In real world, however such behaviour is difficult to observe. The relative narrow sizes of streamer channel and electrode gap make diffusion enter the game relatively early after only some ms. A more realistic modelization is therefore provided in the following pictures, with a more extended reaction dataset and where again neutral as well charged species densities are displayed as a function of time on a log-log scale. As for the neutral species, during the time length needed for streamer development mainly atomic $\mathrm{N}$ and $\mathrm{O}$ species are created, from electron impact-dissociation (the former more than the latter at such an electron temperature). NO production is quite fast and its density approaches $0.02 \%$ of $\mathrm{N}$ atoms one by the end of the pulse. Ozone formation is slower but almost catches up $\mathrm{NO}$. On the other hand $\mathrm{NO}_{2}, \mathrm{NO}_{3}$ and $\mathrm{N}_{2} \mathrm{O}$ production is clearly much slower and become appreciable only on a larger scale time, even more so for $\mathrm{N}_{2} \mathrm{O}_{5}$. After the pulse end, in the temporal range from 2 to $20 \mathrm{~ns}, \mathrm{O}$ and $\mathrm{NO}$ slowly increase till saturation is reached. $\mathrm{N}$ density reaches a value of $9 \times 10^{15} \mathrm{~cm}^{-3}$, whereas $\mathrm{O}$ grows to $3 \times 10^{15} \mathrm{~cm}^{-3}$ and $\mathrm{NO}$ to a value of $4 \times 10^{13} \mathrm{~cm}^{-3}$. The dissociation degree is therefore rather limited, up to $5 \times 10^{-4}$. Ozone, on the contrary, keeps increasing eventually reducing $\mathrm{O}$ and $\mathrm{NO}$ and peaking afterwards. Starting from $0.1 \mathrm{~ms}$, the diffusion term becomes relevant and all species density starts to decrease. The gas phase is practically cleared out after a few ms. Ozone density exceeds atomic oxygen after about $10 \mu \mathrm{s}$, at a level of $1.3 \times 10^{15} \mathrm{~cm}^{-3}$, and peaks after $30 \mu \mathrm{s}$, at a level of $2 \times 10^{15} \mathrm{~cm}^{-3}$ about $100 \mathrm{ppm}$. The increase is triggered by the partial balancing between production from $\mathrm{O}+\mathrm{O}_{2}$ and loss due to dissociative attachment

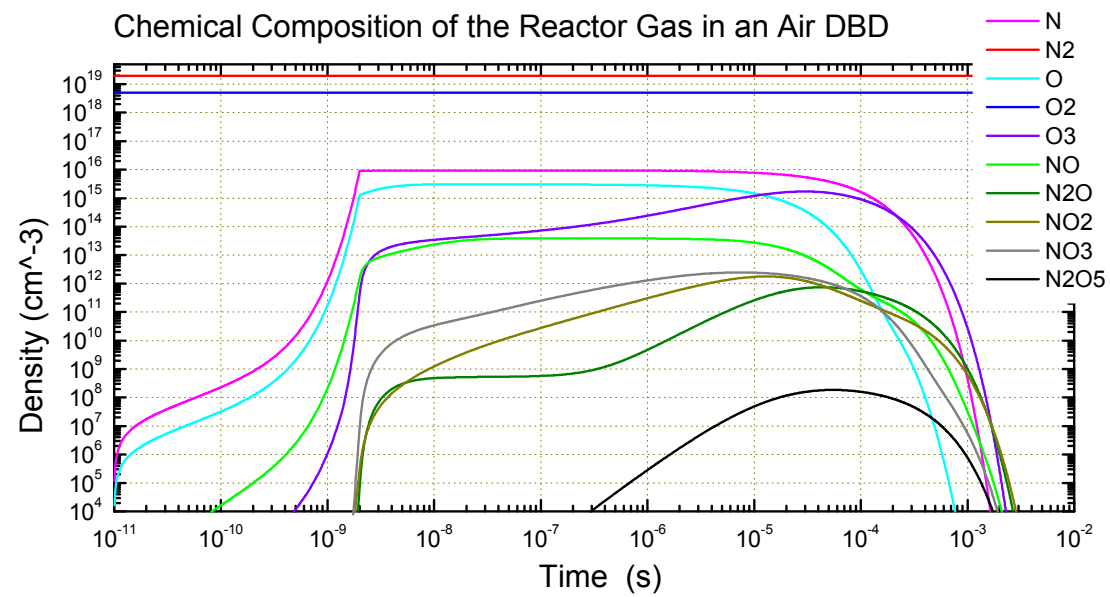

Fig. 3.2.3. Time evolution of the neutral species density in the gas-phase of a streamer in an air DBD. After a brief transient due to streamer formation, the neutral gas-phase reaches a definite composition in about $0.1 \mathrm{~ms}$ and it is then dissipated by diffusion in a few $\mathrm{ms}$. 


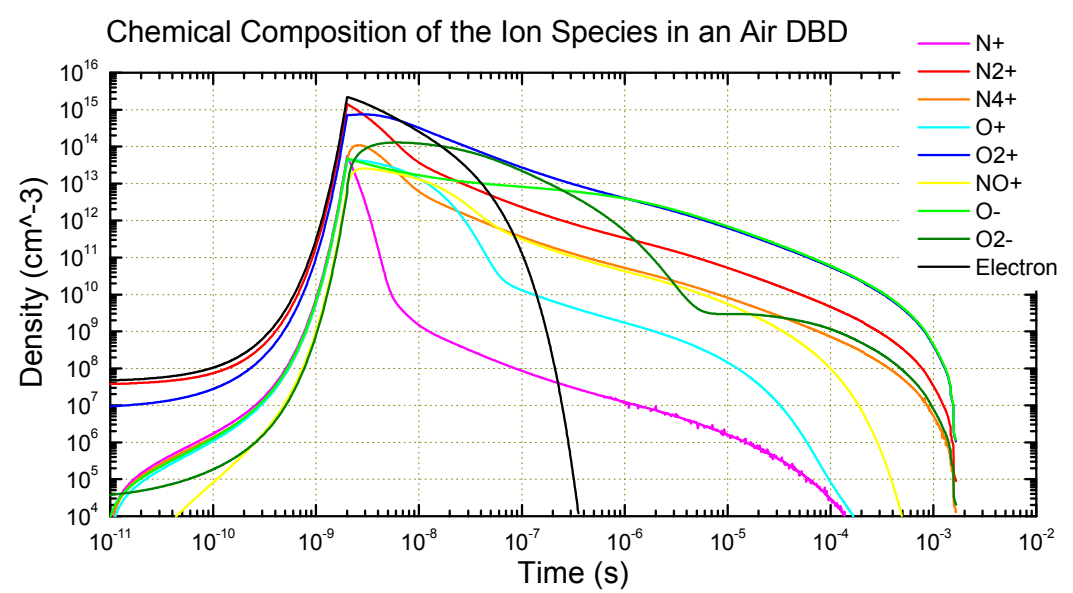

Fig. 3.2.4. Time evolution of the charged species density in the gas-phase of a streamer in an air DBD. After the transient producing an almost exponential increase of charged particles, electrons are quickly removed and an electron-less cold plasma is slowly dissipated due to recombination and then diffusion in about $10 \mathrm{~ms}$.

and charge exchange reactions. Since charged particles are destroyed faster than neutrals due to the high rate of ion-ion recombination, ozone keeps on growing, until atomic $\mathrm{O}$ is finished and then declines due to diffusion. $\mathrm{NO}_{2}$ and $\mathrm{NO}_{3}$ appear to follow a similar trend, with density three order of magnitude lesser and a peak value after tens of $\mu \mathrm{s}$, at a level of $2 \times 10^{12} \mathrm{~cm}^{-3} \cdot \mathrm{N}_{2} \mathrm{O}_{5}$ also has a similar trend, with much lower density and a slight delay. three order of magnitude lesser and a peak value after tens of $\mu$ s, at a level of $2 \times 10^{12} \mathrm{~cm}^{-3}$. On the other hand, $\mathrm{N}_{2} \mathrm{O}$ shows a partial equilibrium within a hundred of ns, then it grows to levels similar to the other nitrogen oxides. Its dynamics is closely controlled by fast dissociative attachment and charge exchange reactions in particular on atomic oxygen ions. So its concentration cannot rise until most of the electrons are removed. $\mathrm{N}_{2} \mathrm{O}$ density appears to take over $\mathrm{NO}_{2}$ after $100 \mu$ s and reaches a maximum of $7 \times 10^{11} \mathrm{~cm}^{-3}$ soon later.

On the other hand, the temporal dynamics of charged species seems to be faster than that of neutrals. This is due to the higher rates of ion-ion recombination and of electron attachment. All the charged species increase almost exponentially during the streamer development time ( $2 \mathrm{~ns})$ and then the total charge density begins to decrease because of recombination. In presence of oxygen, after the pulse ends, when the electron temperature decreases to less than $2 \mathrm{eV}$, electrons are quickly removed due to attachment processes. $\mathrm{O}^{-}$is the majority negative ion, except for a small time window between 10 and 300 ns. This happens because, while at $\mathrm{T}_{\mathrm{e}}=4 \mathrm{eV}$ the dominant attachment process on oxygen is dissociative to $\mathrm{O}^{-}$, at set temperature prevails direct attachment to $\mathrm{O}_{2}$ - Then electrons disappear and soon after charge exchange reactions on oxygen atoms removes $\mathrm{O}_{2}-$ and produces again the more stable $\mathrm{O}$ - Positive charged species composition is mainly determined by charge exchange reactions on $\mathrm{N}_{2}$ and $\mathrm{O}_{2}$. They quickly remove atomic ions and leave $\mathrm{O}_{2}{ }^{+}$as the majority ion, with a $10 \%$ of $\mathrm{N}_{2}{ }^{+}$and a few $\%$ of $\mathrm{N}_{4}{ }^{+}$and $\mathrm{NO}^{+}$. This situation is reached in about $100 \mathrm{~ns}$. Later on positive charged species density shows the same course: a power-law decrease in time (that is linear on the log-log plot) dictated by the ion-ion recombination process, followed by an exponential decay due to ambipolar diffusion after $0.5 \mathrm{~ms}$. In order to study 
the influence of the different streamer parameters we have performed several simulations changing their values in a broad range. The overall temporal evolution appears to be similar to the one reported in figures, even if some particulars are affected. In next figures the maximum density of a few neutral species is displayed as a function of the peak electron temperature. We made simulations varying Te from 2 to $4.5 \mathrm{eV}$ with a step of $0.25 \mathrm{eV}$. We found that a minimum electron temperature of $2.5 \mathrm{eV}$ is needed in order to allow electron density to rise during the streamer formation time. This can be understood since attachment prevails on ionization at small electron temperatures. The general behaviour shows that the maximum density decreases by increasing the electron temperature. This is due to the fact that ionization rate rises faster than the dissociation rate as the electron temperature is increased. Indeed, those simulations were performed keeping the peak electron density constant and since it means that the ionization grade stays constant then the overall dissociation grade should be diminished. The effects are more pronounced for species produced by secondary processes after the streamer formation, such as $\mathrm{O}_{3}, \mathrm{NO}_{2}$ and $\mathrm{N}_{2} \mathrm{O}$ than for atomic radicals such as $\mathrm{N}$ and $\mathrm{O}$. We also checked that the length of the electric field pulse does not make any significant difference in a broad range from 0.2 to $5 \mathrm{~ns}$. Indeed most of the chemical kinetics is simply frozen during such fast times and the total production of species through electron impact reactions during the pulse depends on the electron temperature (through the reaction rates) and on the electron density which is reached at the end of the pulse rather than on the pulse length. We have also performed several simulations changing the peak electron density ne from $10^{11} \mathrm{~cm}^{-3}$ to $10^{16} \mathrm{~cm}^{-3}$. Again, the temporal dynamics appears to be similar, with the same behavior of nitrogen oxides, ozone and atomic radicals and ions. In figure the maximum density of a few neutral species is displayed as a function of the peak electron density. All the maximum densities appear to increase increasing the electron density. The increase is approximately linear on the log-log scale of the figure. However the effect is much more pronounced for the heavier nitrogen oxides, thus increasing their concentration in the late gas phase, where ozone percentage in excess of $1000 \mathrm{ppm}$ is reported. The strong sensitivity to such a badly known parameter of the discharge is clearly

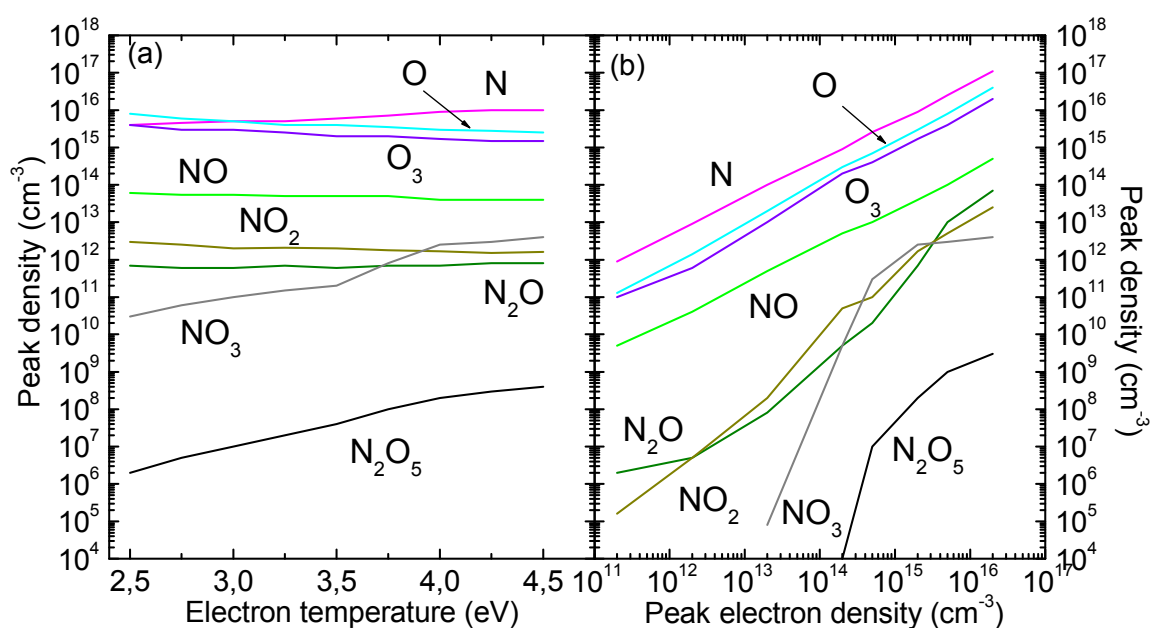

Fig. 3.2.5. Dependence of the peak density reached by different neutral species on the electron temperature and maximum density in the gas-phase of a streamer in an air DBD. 
disappointing from the point of view of driving the optimization process with chemical kinetics modeling, but it offers also a clue for an indirect insight of the streamer properties from this strong link between $\mathrm{n}_{\mathrm{e}}$ and the composition of radical fluxes outside the streamer. We have also investigated the effects due to the streamer radius Rs. Such a parameter affects mainly the diffusion process. We have varied Rs between 0.05 to $1 \mathrm{~mm}$. The smaller Rs is, the faster diffusion transports radicals and ions outside the streamer. If it happens fast enough it interferes with secondary chemical processes leading to transformation of atomic radicals in $\mathrm{O}_{3}, \mathrm{NO}_{2}$ and $\mathrm{N}_{2} \mathrm{O}$. A slower diffusion gives time to the system to approach the chemical equilibrium in composition. However equilibrium is approached in times exceeding a few hundreds milliseconds, which is definitely too long respect to any reasonable diffusion time.

\subsection{Reactivity of different nitrogen oxides}

Plasma remediation is an efficient and promising technology to destroy toxic and greenhouse gases (Manheimer, 1997). These techniques are potentially lower in cost and more efficient than conventional thermal incineration. In these plasmas, the majority of electrical energy is expanded in heating electrons, rather than heating gas, as discussed previously. The understanding of the process and the optimization of the efficiency benefit largely from computational studies of the dynamics of the plasma gas-phase. In particular the air chemical kinetics of high-pressure dielectric barrier discharges. Such systems are considered in the context of volatile organic compound (VOC) and NOx remediation. The air plasma kinetic processes are discussed with the goal of providing insight for optimizing efficiencies. Indeed the most extensively investigated applications of this type are used to treat dilute concentrations of toxic molecules in airstreams. Potential advantages of the highpressure, nonthermal plasma approach include the highly energy efficient selectivity provided by the plasma chemistry and its capability for minimizing secondary-waste production. Atmospheric pressure operation is preferred for high-throughput waste processing (Shoyama, 2007). Indeed the removal of NO from environment is a relevant problem concerning pollution control (Amoroso, 2008). NO stays in the low atmosphere for several days and enters cycles producing ozone, causing smog. Nitrogen oxides are mainly produced as by-product in combustion processes in free air. For instance in diesel engine exhaust NO can reach 200-500 ppm and $\mathrm{NO}_{2} 50$ ppm (Srinivasan, 2007). So we applied our simulations to air contaminated by similar level of nitrogen oxides. In Figures 3.3.1 and 3.3.2 we display the results of a simulation, with the same standard parameters but starting with $500 \mathrm{ppm}$ of NO, that is a density of $1.3 \times 10^{16} \mathrm{~cm}^{-3}$. No diffusion of neutrals is considered here, to let the system reach the chemical equilibrium, which however happens only at late times, both considering discharge repetition times (several $\mathrm{kHz}$ generally) and diffusion times for the considered streamer radius (a few ms). After the end of the discharge at 2 ns, NO is completely removed, oxidated initially by $\mathrm{O}$ atoms and then, when atomic radicals disappear, consumed in reactions with ozone and $\mathrm{NO}_{2}$. In the final state it is substituted mainly by ozone (about $1000 \mathrm{ppm}$ ).

A substantial amount of $\mathrm{NO}_{2}(192 \mathrm{ppm})$ and $\mathrm{N}_{2} \mathrm{O}(11 \mathrm{ppm})$ is produced too, while other oxides are not formed with an appreciable concentration. The transition from oxygen atoms to ozone happens after about $10 \mu \mathrm{s}$, while NO disappears only after $10 \mathrm{~ms}$. More details are discussed in literature (Barni, 2010). Starting from $\mathrm{NO}_{2}$ as initial contaminant produces a different kinetic pattern. Oxidation and substitution with ozone in the final state is achieved 


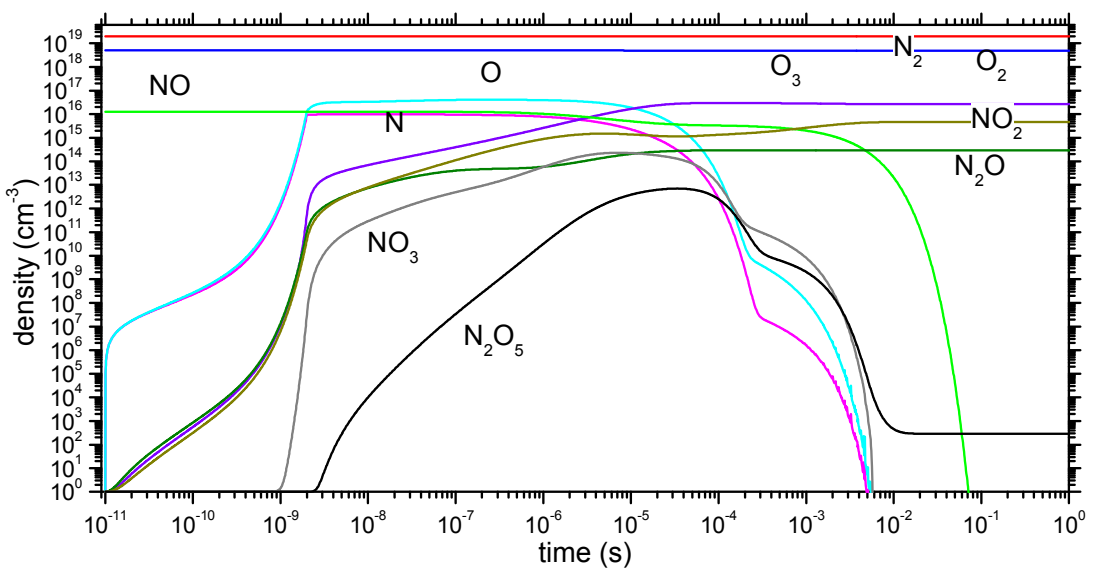

Fig. 3.3.1. Time evolution of the neutral species density in the gas-phase of a streamer. NO is removed to mainly ozone, the transition happening within a few tens of microseconds.

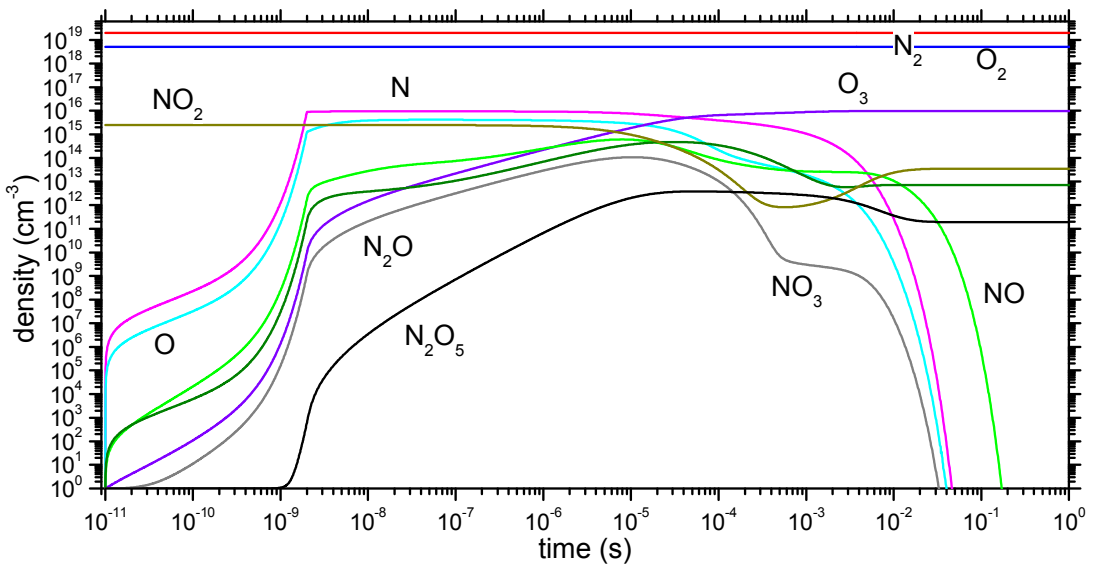

Fig. 3.3.2. Time evolution of the neutral species density in the gas-phase of a streamer. $\mathrm{NO}_{2}$ is removed to mainly ozone, the transition happening within a few tens of microseconds.

too, but the initial nitrogen oxide is not completely removed and about $2 \%$ is not disposed. A similar trend is reported for $\mathrm{N}_{2} \mathrm{O}$ which is however better oxidised.

The effect of the repetition of streamer discharges during subsequent cycles of a.c. voltage was studied and displayed in Figure 3.3 .4 at $10 \mathrm{kHz}$. A small number of discharges is needed to remove NO under a few \%. To reach an equilibrium in the gas-phase, here producing a constant flux of ozone, consuming mainly atmospheric oxygen, requires a larger number of cycles, in the order of thousands, yet in times less than seconds. In this case substantial amounts of radicals in the form of atomic nitrogen and oxygen are present and could be delivered to materials exposed to the plasma phase, for instance to achieve oxidation of biological matter for sterilization purposes (Moreau, 2008). 


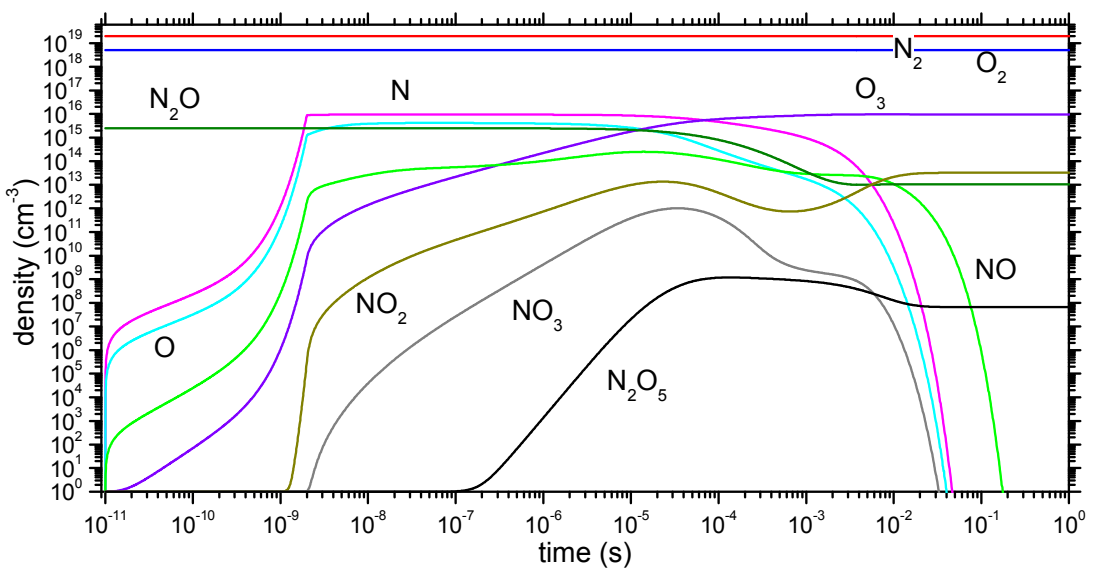

Fig. 3.3.3. Time evolution of the neutral species density in the gas-phase of a streamer. $\mathrm{N}_{2} \mathrm{O}$ is removed to mainly ozone, the transition happening within a few tens of microseconds.

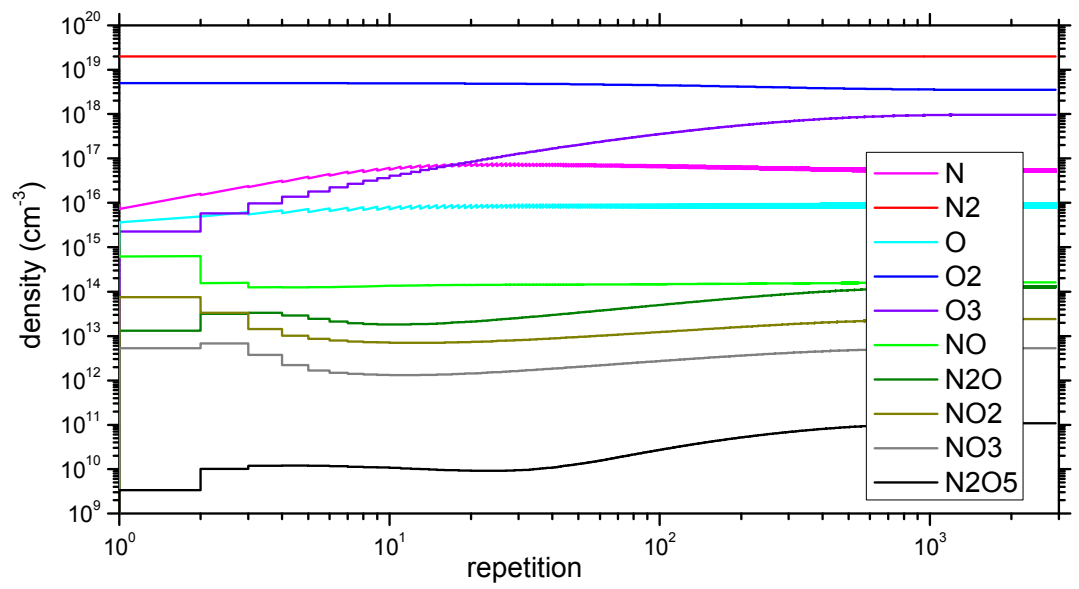

Fig. 3.3.4. Evolution of the neutral species density in the gas-phase of a streamer under subsequent repetitions at a frequency of . $\mathrm{N}_{2} \mathrm{O}$ is removed to mainly ozone, the transition happening within a few tens of microseconds.

\subsection{Effect of gas temperature}

Reaction rates for ion as well as neutral species depends on their temperature, which could depart from the set, that is $300 \mathrm{~K}$. Many neutral gas reactions are quite affected by the gas temperature, so it is interesting to use the theoretical framework we have developed to study what is the impact of a hotter gas phase. In any case, since thermal energy is much smaller than the molecular bond energy, the initial step in the decomposition is presumed to involve again mostly electron-impact dissociation. An idea of the impact the gas temperature has on the chemical kinetics is reported in figure. Taking into account the air 
temperature within the device to be simulated, the software has been changed adding the temperature dependence in several reactions involving neutral species, rates have been collected through different public databases as coefficients for the Arrhenius equation. The main results was that at low temperature it has been observed a cut down in nitrogen monoxide concentrations, which has been replaced by ozone as principal product. At high temperature nitrogen monoxide remains unperturbed through the discharge while ozone disappears. Thus gas phase temperature, and then the control of a strictly cold plasma condition is crucial for efficient NOx remediation.

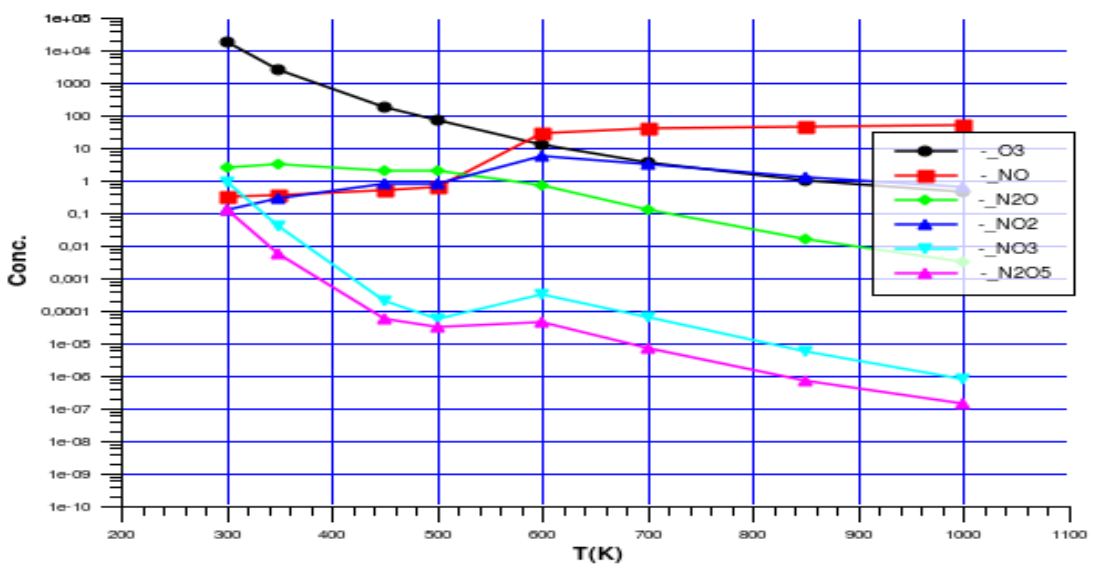

Fig. 3.4.1. Final composition of the neutral species density in the gas-phase of a streamer. $\mathrm{NO}$ is removed to ozone only at low temperatures, the transition happening at about $600 \mathrm{~K}$.

\section{Chemical kinetics in air steady plasmas}

Then we consider application of the modeling to a steady plasma conditions, like the one in a corona discharge. In order to study direct chemical kinetics effect we have chosen an ideal setup, under a well-mixed reactor approximation, and with an uniform plasma background.

Apart from geometry (here $3 \mathrm{~mm}$ corona electrode is discussed), we have parametrised our results in terms of two physical values, the effective electron density and temperature. As discussed previously, the former affects mostly the velocity of the chemical kinetics evolution, whereas the latter affects the relative weight of the different electron impact. In order to study the influence of the different parameters we have performed several simulations changing their values in a broad range. The overall temporal evolution appears to be similar to the one reported in figure, even if some particulars are affected. The corona discharge produce mainly NO reaching a steady state after a few hundreds of microseconds. There is a transition from the initial phases of the discharge, where $\mathrm{N}$ and NO are the major neutrals. All neutral molecules are accumulating in the gas-phase until 10 microseconds. Then nitrogen atoms start to decline, followed a little after by ozone. The final composition is then reached, with about $90 \%$ of $\mathrm{NO}$ as final product. In next figure the maximum density of a few neutral species is displayed as a function of the peak electron temperature and 
density. We made simulations varying Te from 0.5 to $2 \mathrm{eV}$ with a step of $0.25 \mathrm{eV}$. We found that above $1 \mathrm{eV}$ the density of nitrogen oxides produced in the gas-phase becomes independent from the temperature. Ozone and atomic radicals are minority showing a broad minimum around $1 \mathrm{eV}$. We have also performed several simulations changing the peak electron density ne from $10^{8} \mathrm{~cm}^{-3}$ to $10^{12} \mathrm{~cm}^{-3}$. Again, the temporal dynamics appear to be similar, with the same behavior of nitrogen oxides, ozone and atomic radicals and ions.

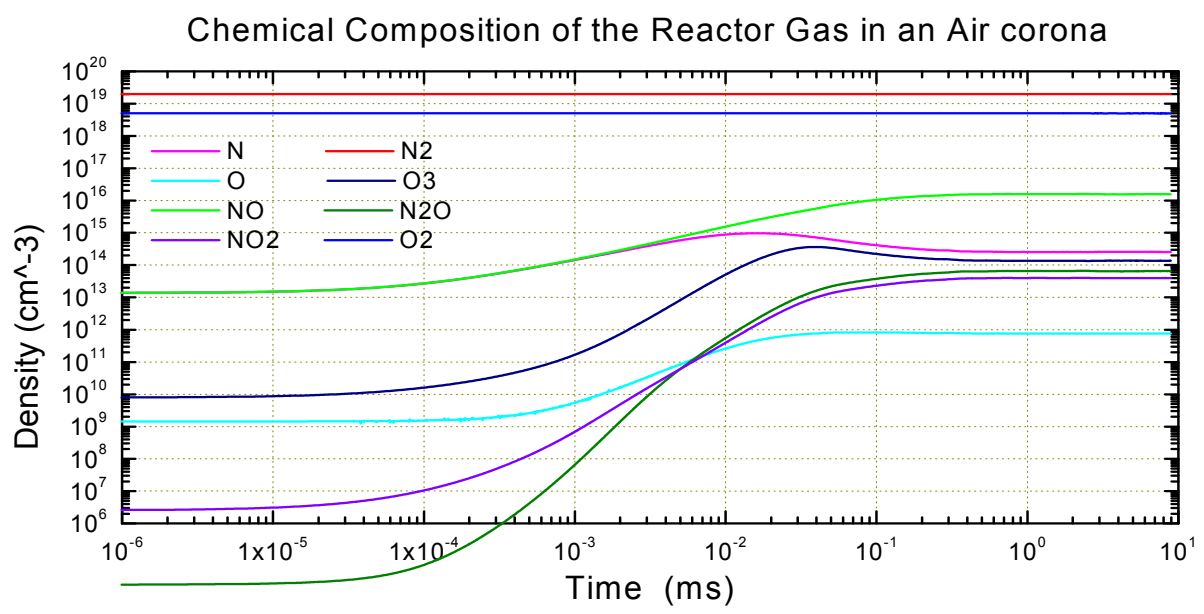

Fig. 4.1. Time evolution of the neutral species density in the gas-phase of an air corona. The neutral gas-phase reaches an equilibrium in about one second, the transition happening between 1 and $100 \mathrm{~ms}$.

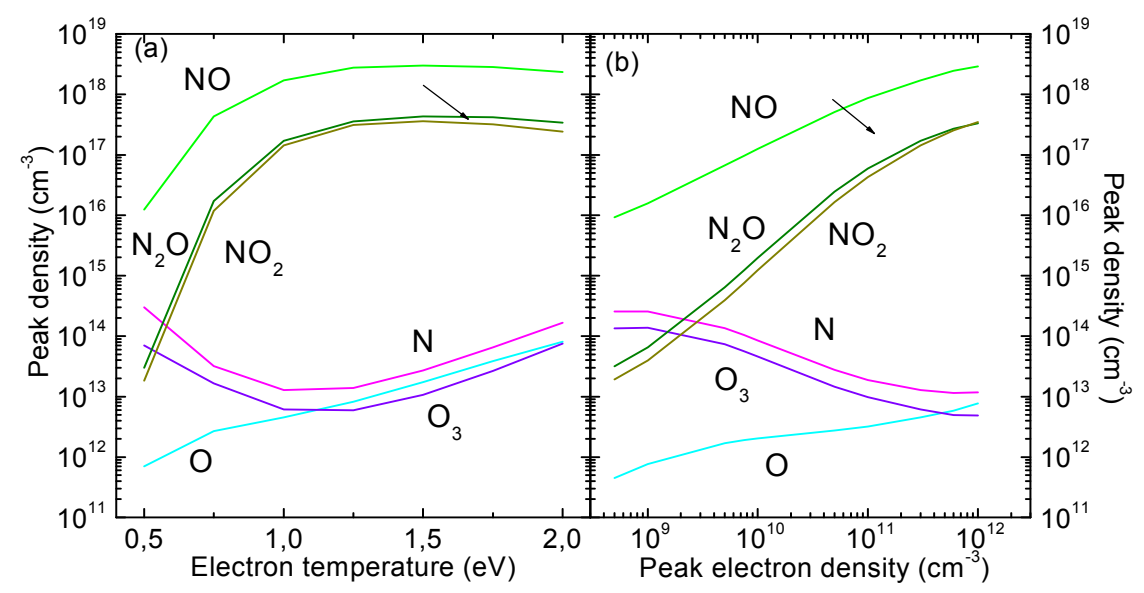

Fig. 4.2. Dependence of the peak density reached by different neutral species on the electron temperature and density in the gas-phase of an air corona.

All the nitrogen oxides densities appear to increase increasing the electron density. The increase is approximately linear on the log-log scale of the figure, showing a trend to 
saturation at the higher end of the range. The effect is much more pronounced the heavier nitrogen oxides, thus increasing their concentration in the late gas phase. Ozone and nitrogen atoms decrease, becoming comparable to atomic oxygen.

\section{Conclusions}

A chemical kinetics modeling of atmospheric pressure air discharges in the streamer regime of DBDs as well as in corona steady state plasmas has been presented. The model was used to evaluate the prospect of nitrogen oxides removal from the gas phase. The results obtained show that the chemical system could remove $\mathrm{NO}$ and to some extent also other nitrogen oxides up to a certain maximum initial concentration. Under realistic conditions for NO this could be as high as $800 \mathrm{ppm}$. The system produces mainly ozone through secondary reactions in the gas-phase. This theoretical study shows that important and significant aspects determined by the chemical kinetics effects could be investigated through simulations. Then they could prove worthy in order to study the applications of plasmas at high pressure in streamer regime (for example DBDs) and their capabilities as gas processing units.

\section{Acknowledgment}

The authors are deeply grateful to their collaborators and students that over several years have partecipated in their research studies, in the laboratory activities and in discussion about plasma science during working hours and not, contributing to the success of the PlasmaPrometeo Center of Excellence. We could enlist M.Piselli, S.Zanini, P.Esena, I.Nardi, E.Grimoldi, F.Fumagalli, I.Biganzoli just to mention a few involved in the topics of this chapter.

\section{References}

Amoroso, A.; Beine, H.J.; Esposito, G.; Perrino, C.; Catrambone, M. \& Allegrini, I. (2008). Water Air and Soil Pollution, Vol. 188, pp. 81

Barni, R. \& Riccardi, C. (2010). Perspective of NOx removal from numerical simulation of non-thermal atmospheric pressure plasma chemical kinetics, High Temperature Material Processes, Vol. 14, pp. 205-210

Barni, R.; Esena, P. \& Riccardi, C. (2005). Chemical kinetics simulation for atmospheric pressure air plasmas in a streamer regime, Journal of Applied. Physics, Vol. 97, pp. 073301.1-7

Barni, R.; Esena, P. \& Riccardi, C. (2005). Chemical kinetics simulations of an atmospheric pressure plasma device, Surface \& Coatings Technology, Vol. 200/1-4, pp. 924-927

Benson, S.W. (1982). Thermochemical Kinetics, Wiley, New York US

Braun, D.; Gibalov, V.I. \& Pietsch, G.J. (1991). Journal of Physics D, Vol. 24, pp. 564

Chantry, P.J. (1987). Journal of Applied. Physics, Vol. 62, pp. 1141

Esena, P.; Riccardi, C.; Zanini, S.; Poletti, G. \& Orsini, F. (2005). Surf. E Coatings Techn, Vol. 1/4, pp. 664

Guerra, V. \& Louriero, J. (1997). Plasma Sources Science E Technology, Vol. 6, pp. 373

Kogelschatz, U. (2003). Plasma Chemistry and Plasma Processing, Vol.23, pp.1 
Kossyi, I.A.; Kostinsky, A.Y.; Matveyev, A.A. \& Silakov, V.P. (1992). Kinetic scheme of the non-equilibrium discharges in nitrogen-oxygen mixtures, Plasma Sources Science $\mathcal{E}$ Technology, Vol. 1, pp. 207-220

Kulikovsky, A.A. (1997). Journal of Physics D, Vol.30, pp.441

Kulikovsky, A.A. (1998). Physical Review E, Vol.57, pp.7066

Lichtenberg, A.J. ; Lieberman, M.A. ; Kouznetsov, I.G. \& Chung, T.H. (2000). Plasma Sources Science E Technology, Vol. 9, pp. 45

Malik, D.A. ; Orlov, K.E. ; Miroshnikov, I.V. \& Smirnov, A.S. (2008). Spatial and temporal evolution of a dielectric barrier discharge, Journal of Applied Physics, Vol. 103, pp. 033303

Manheimer, W.; Sugiyama, L.E. \& Stix, T.H. (1997). Plasma Science and the Environment, American Institute of Physics, Woodbury, NY US

Mariotti, D \& Sankaran, R.M. (2010). Microplasmas for nanomaterials synthesis, Journal of Physics D, Vol.43, pp.323001.1-21

Moreau, M. ; Orange, N. \& Feuilloley, M.G. (2008). Non-thermal plasma technologies: new tools for biodecontamination, Biotechnology Advances, Vol. 26, pp. 610

Raizer, Y.P. (1991). Gas Discharge Physics, Springer-Verlag, Heidelberg Germany

Raffaele-Addamo, A.; Riccardi, C.; Selli, E.; Barni, R.; Piselli, M.; Poletti, G.; Orsini, F.; Marcandalli, B.; Massafra, M.R. \& Meda, L. (2003). Surf. E Coatings Techn, Vol. 1745, pp. 886

Riccardi, C.; Barni, R.; De Colle, F. \& Fontanesi, M.(2000). IEEE Trans. on Plasma Science, Vol. 28, pp. 278

Riccardi, C.; Barni, R. \& Fontanesi, M. (2001). Journal of Applied. Physics, Vol. 90, pp. 3735

Shoyama, T. \& Yoshioka, Y. (2007). Theoretical study of methods for improving the energy efficiency of NOx removal from diesel exhaust gases by silent discharge, Electrical Engineering in Japan, Vol. 161, pp. 1-9

Siliprandi, R.A.; Roman, H.E.; Barni, R. \& Riccardi, C. (2008). Characterization of the streamer regime in dielectric barrier discharges, Journal of Applied Physics, Vol. 104, pp. 063309.1-9

Srinivasan, A.D. \& Rajanikanth, B.S. (2007). IEEE Transactions on Industrial Applications, Vol. 43, pp. 1507 


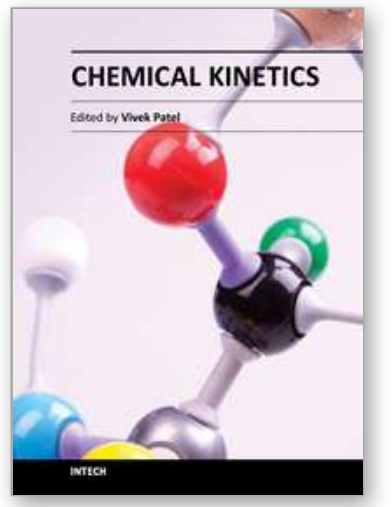

\author{
Chemical Kinetics \\ Edited by Dr Vivek Patel
}

ISBN 978-953-51-0132-1

Hard cover, 344 pages

Publisher InTech

Published online 29, February, 2012

Published in print edition February, 2012

Chemical Kinetics relates to the rates of chemical reactions and factors such as concentration and temperature, which affects the rates of chemical reactions. Such studies are important in providing essential evidence as to the mechanisms of chemical processes. The book is designed to help the reader, particularly students and researchers of physical science, understand the chemical kinetics mechanics and chemical reactions. The selection of topics addressed and the examples, tables and graphs used to illustrate them are governed, to a large extent, by the fact that this book is aimed primarily at physical science (mainly chemistry) technologists. Undoubtedly, this book contains "must read" materials for students, engineers, and researchers working in the chemistry and chemical kinetics area. This book provides valuable insight into the mechanisms and chemical reactions. It is written in concise, self-explanatory and informative manner by a world class scientists in the field.

\title{
How to reference
}

In order to correctly reference this scholarly work, feel free to copy and paste the following:

Claudia Riccardi and Ruggero Barni (2012). Chemical Kinetics in Air Plasmas at Atmospheric Pressure, Chemical Kinetics, Dr Vivek Patel (Ed.), ISBN: 978-953-51-0132-1, InTech, Available from: http://www.intechopen.com/books/chemical-kinetics/chemical-kinetics-in-air-plasmas-at-atmospheric-pressure

\section{INTECH}

open science | open minds

\author{
InTech Europe \\ University Campus STeP Ri \\ Slavka Krautzeka 83/A \\ 51000 Rijeka, Croatia \\ Phone: +385 (51) 770447 \\ Fax: +385 (51) 686166 \\ www.intechopen.com
}

\author{
InTech China \\ Unit 405, Office Block, Hotel Equatorial Shanghai \\ No.65, Yan An Road (West), Shanghai, 200040, China \\ 中国上海市延安西路65号上海国际贵都大饭店办公楼405单元 \\ Phone: +86-21-62489820 \\ Fax: $+86-21-62489821$
}


(C) 2012 The Author(s). Licensee IntechOpen. This is an open access article distributed under the terms of the Creative Commons Attribution 3.0 License, which permits unrestricted use, distribution, and reproduction in any medium, provided the original work is properly cited. 